\title{
A Study on the Preparation and Characterization of Curcumin and Gold Nanorods-loaded Albumin Nanoparticles and its Treatment of MCF-7 Breast Cancer Cells in Vitro
}

\author{
Chen LIANG ${ }^{1, a}$, Hao ZHANG ${ }^{1, b}$, Lin WANG ${ }^{1, c}$, Dong-Sheng ZHANG ${ }^{1, d, *}$ \\ ${ }^{1}$ Medical School of Southeast University, Jiangsu Key Laboratory For Biomaterials and Devices, \\ Nanjing, Jiangsu Province, China \\ ac122836@163.com, 'bhanghao_112_sh@126.com, cw1031995@163.com, \\ dzdszds1222@163.com \\ ${ }^{*}$ Corresponding author
}

Keywords: Curcumin and Gold Nanorods-loaded Albumin Nanoparticles, Photothermal Therapy, Breast Cancer.

\begin{abstract}
This work aims to study the preparation and characterization of curcumin and gold nanorods-loaded albumin nanoparticles and investigate its treatment for MCF-7 human breast cancer cells in vitro. Gold nanorods (GNRs) were prepared by seedless method. While the curcumin and gold nanorods-loaded albumin nanoparticles (Cur/GNRs-BSA-NPs) were prepared by desolvation-crosslinking method, with the characteristics detected by dynamic light scattering (DLS), transmission electron microscope (TEM) and ultraviolet photometer. The results indicated that the GNRs and Cur/GNRs-BSA-NPs were prepared successfully. The prepared GNRs, which are rectangular and round-ended, have uniform sizes and good dispersion. The Cur/GNRs-BSA nanoparticles were spherical with exact average diameter of 190nm. The Cur/GNRs-BSA-NPs did not show cytotoxicity in vitro after tested by the CCK-8 assay. Thermodynamic test was employed to observe the temperature change of various doses of Cur/GNRs-BSA-NPs at the near-infrared (NIR) light exposure with the wavelength of $808 \mathrm{~nm}$ and the output power of $2.5 \mathrm{~W}$. It was found that the Cur/GNRs-BSA-NPs induced significant temperature elevation and curcumin release upon irradiation with NIR light. The anti-tumor effect on MCF-7 human cancer cells treated with Cur/GNRs-BSA-NPs in vitro was evaluated by CCK-8 assay. The experimental results revealed that Cur/GNRs-BSA-NPs could significantly inhibit the proliferation of MCF-7 cells with combined treatment. Moreover, the coating of albumin could help reduce toxicity of GNRs and improve the bioavailability of the insoluble curcumin. The Cur/GNRs-BSA-NPs developed in this work present a new potential method combining both traditional Chinese and western medical treatment for human breast cancer.
\end{abstract}

\section{Introduction}

Breast cancer in women is a major public health problem throughout the world. Even though many treatments,such as surgery, radiation and drug therapy, have been developed for breast cancer. There is still a need for multi-functional cancer treatment which maximizes therapeutic effects through less invasive techniques. A hybridization of effective treatments could offer combinational therapy, which may result in higher success rates and increased quality of life for patients suffering from the disease.

Nanoparticles show properties that are often different from those of their corresponding bulk materials. Besides the specific physical and chemical properties, Gold nanorods (GNRs) have the ability to absorb relatively harmless near infrared (NIR) light, causing the subsequent release of energy via heat, which make them particularly attractive tools for cancer hyperthermia therapy [1]. Curcumin, a traditional Chinese medical ingredient with poor solubility and stability, has been demonstrated to possess a broad range of medicative properties, including anti-inflammatory, anti-cancer, anti-oxidant, and chemopreventive activities[2]. However, the clinical application is limited by its main drawback including instability, low solubility, and poor bioavailability [3]. By a 
targeting-delivery system, drugs can be delivered and concentrated in a local district such as target tissues, organs, cells, or a special district of cells. Albumin, an omnipotent protein carrier for drug delivery, has been proven to be non-immunogenic, nontoxic, biodegradable, and biocompatible [4]. Due to the specific structure of amino acids in the albumin molecule, albumin is a suitable matrix material for fabricating nanoparticles (NPs) for drug delivery.

In this work, we prepared GNRs with a simple and efficient synthesize method and proved that the GNRs could be successfully encapsulated within the Cur-BSA. When exposure to the NIR light, the formed Cur/GNRs-BSA-NPs have been demonstrated to induced significant temperature elevation and then gently rise to a steady temperature ranging from $38^{\circ} \mathrm{C} \sim 49^{\circ} \mathrm{C}$ depending on the its different concentration. CCK-8 assay results also indicated that the nanoparticles showed combined therapeutic treatment on the human breast cancer MCF-7 cells in vitro and significantly inhibit the proliferation of the cancer cells.

\section{Materials and Methods}

\section{Materials}

Hexadecyl trimethyl ammonium bromide (CTAB); Chloroauric acid $\left(\mathrm{HAuCl}_{4} \cdot 4 \mathrm{H} 2 \mathrm{O}\right)$; Sodium borohydride $\left(\mathrm{NaBH}_{4}\right)$; Silver nitrate $\left(\mathrm{AgNO}_{3}\right)$; Bovine serum albumin (BSA); Curcumin; Hydroquinone $\left(\mathrm{C}_{6} \mathrm{H}_{4}(\mathrm{OH})_{2}\right)$; Sodium hydroxide $(\mathrm{NaOH})$. All reagents used were commercially available and of analytical grade.

\section{Synthesis of GNRs Solution}

The GNRs were prepared by a simple and efficient seedless synthesis method [5]. Briefly, $3.6 \mathrm{~mL}$ of $0.01 \mathrm{~mol} / \mathrm{L} \mathrm{HAuCl} \mathrm{H}_{4} \cdot 4 \mathrm{H}_{2} \mathrm{O}$ was mixed with $85.2 \mathrm{~mL}$ of $0.1 \mathrm{~mol} / \mathrm{L} \mathrm{CTAB}$ solution in a beaker (200 $\mathrm{mL}$ ). Then, $0.5 \mathrm{~mL}$ of $0.02 \mathrm{~mol} / \mathrm{L} \mathrm{AgNO}_{3}$ aqueous solution was added. The resulting seed solution became bright yellow color. Subsequently, $1.44 \mathrm{~mL}$ of $0.33 \mathrm{~mol} / \mathrm{L} \mathrm{C}_{6} \mathrm{H}_{4}(\mathrm{OH})_{2}$ aqueous solution were added under rapid mixing, which changed the color from yellow to colorless. After that, $0.5 \mathrm{~mL}$ of ice-cold $0.5 \mathrm{mmol} / \mathrm{L} \mathrm{NaBH}_{4}$ was added with vortex mixing for $2 \mathrm{~min}$. As the final step, the mixed solution in the beaker was incubated in a dark water bath kettle with temperature about $28 \sim 30{ }^{\circ} \mathrm{C}$ for $12 \mathrm{~h}$ overnight. As the reaction finished, the excess CTAB surfactant was discarded from the final product by centrifugation at $13500 \mathrm{rpm}, 20 \mathrm{~min}$ and then the precipitate was re-dispersed in $1 \mathrm{~mL}$ of distilled and de-ionized (DI) water for three times.

\section{Preparation of Cur-BSA-NPs, GNR-BSA-NPs, Cur/GNRs-BSA-NPs}

Cur-BSA-NPs were prepared using a desolvation method, followed by cross-linking with glutaraldehyde [6]. Briefly, $50 \mathrm{mg}$ of bovine serum albumin (BSA) was dissolved in $4.0 \mathrm{~mL}$ of water. This aqueous phase was then desolvated with $4 \mathrm{~mL}$ of ethanol, in which $10 \mathrm{mg}$ of curcumin had been dissolved. Adjusting the $\mathrm{PH}$ of the hybrid solution to about $9.0 \mathrm{using} 1 \mathrm{~mol} / \mathrm{L} \mathrm{NaOH}$, then, under stirring with magnetic stirrer, ethanol was added dropwise to the aqueous phase at a steady rate of 1 $\mathrm{mL}$ per minute. After some while, the coacervates were formed and hardened using $50 \mathrm{uL}$ of $25 \%$ glutaraldehyde.

In a similar way, the GNR-BSA-NPs were prepared, except that $0.5 \mathrm{~mL}$ of the prepared GNRs solution described at above section was mixed in the BSA solution and the $\mathrm{PH}$ of the hybrid solution was adjusted to about 9.0 using $1 \mathrm{~mol} / \mathrm{L} \mathrm{NaOH}$. With the same process, ethanol and glutaraldehyde were used to form and harden the coacervates at gentle stirring overnight.

Preparation of Cur/GNRs-BSA-NPs was achieved by Liangke Zhang protocol [7,8]. Before preparation, the prepared GNRs solution and curcumin dissolved solution above were dispersed with ultrasonic vibrator. $50 \mathrm{mg}$ of BSA was dissolved in $4.0 \mathrm{~mL}$ of water. This aqueous phase was then mixed with $0.5 \mathrm{~mL}$ of the GNRs solution and a little of curcumin solution. $1 \mathrm{~mol} / \mathrm{L} \mathrm{NaOH}$ was added to adjust the $\mathrm{PH}$ to about 9.0, too. Subsequently, with the same process using ethanol and glutaraldehyde like Cur-BSA-NPs preparation, Cur/GNRs-BSA-NPs coacervates were acquired. 
All of the nanoparticles prepared above were centrifuged at $12000 \mathrm{rpm}, 20 \mathrm{~min}$. The remaining excess reagents in the resulting product were removed by washing three times with ultrapure water.

\section{Characterization}

The morphologies of the prepared GNRs, GNRs-BSA-NPs, Cur-BSA-NPs and Cur/GNRs-BSA-NPs were obtained by using H600-transmission electron microscope (TEM) operating at $200 \mathrm{kV}$. Their diameter, mobility, and zeta potential were measured using a Mobius DLS instrument. Samples were diluted with water prior to analysis.

\section{Cell Culture}

Human Breast cancer MCF-7 cells were purchased from Cell Bank, Chinese Academy of Sciences. The cells were maintained in DMEM culture medium supplemented with $10 \%$ fetal bovine serum in a fully humidified atmosphere at $37{ }^{\circ} \mathrm{C}$ with $5 \% \mathrm{CO}_{2}$.

\section{Thermodynamic Test of the Cur/GNRs-BSA-NPs}

A cell well-plate was placed under the vertically mounted near-infrared (NIR) laser and various doses of Cur/GNRs-BSA-NPs suspended in water and ethanol were placed in the wells. The NIR laser, with a wavelength of $808 \mathrm{~nm}$, was turned on at $2.5 \mathrm{~W}$ of output power for up to 60 minutes, while a thermocouple, placed on the side of the wells, recorded the temperature at 5 min intervals.

\section{Therapeutic Experiment on MCF-7 Cells in Vitro}

The experiment was divided into four groups with Cur-BSA-NPs, GNRs-BSA-NPs, and Cur/GNRs-BSA-NPs treated, as well as a negative group untreated. To examine the biological effects of the Cur/GNRs-BSA-NPs on the MCF-7 cancer cells in terms of cell proliferation inhibition rate, CCK-8 assay test was performed. Firstly, MCF-7 cells at logarithmic grow phase were digested with $0.25 \%$ of trypsin and pipetted to the cell suspension. The cell density was adjusted to be $4 \times 10^{4} / \mathrm{ml}$. Then the cells were seeded into 96-well tissue culture plate with $100 \mathrm{ul}$ per well. The cells reached a confluence of $60-80 \%$ after $24 \mathrm{~h}$ incubation. Six wells were prepared for each group. After treated with Cur-BSA-NPs, GNRs-BSA-NPs and Cur/GNRs-BSA-NPs respectively, they were irradiated by NIR laser for 40min and incubated continuously in incubators in a fully humidified atmosphere at $37{ }^{\circ} \mathrm{C}$ with $5 \% \mathrm{CO}_{2}$ for $48 \mathrm{~h}$. $10 \mathrm{ul}$ of CCK-8 solution was added to each well, and the 96-well plate was continuously incubated at the same condition for $40 \mathrm{~min}$, then the OD value for each well was read at wavelength $450 \mathrm{~nm}$ to determine the cell viability on a microplate reader. The cell proliferation inhibition rate was calculated as following:

$$
\text { cell inhibition rate }(\%)=\mathrm{OD}_{(\text {treated experimental group) }} / \mathrm{OD}_{\text {(negative group) }} \times 100 \%
$$

\section{Statistical Analysis}

Data were presented as the mean \pm standard deviation. The statistical significance was determined using the analysis of variance (ANNOVA) and two-tail Student's $t$ test. $P<0.05$ was considered statistically significant.

\section{Results and Discussion}

\section{Characterization of GNRs}

Fig. 1(a) shows a TEM image of the GNRs, which have uniform sizes and good dispersion. The TEM image also illustrates that the preparedt GNRs are rectangular with round-ended morphology. The average transverse diameter of the prepared GNR is about $20 \mathrm{~nm}$ and the longitudinal diameter about $70 \mathrm{~nm}$. Fig. 1(b) displays UV-Vis absorption spectra of the GNR. It is well known that main feature of the absorption spectra for metallic nanoparticle is of the SP resonance band(s). From one up to three SP bands can be observed corresponding to three polarizability axes of the metallic nanoparticles[9]. The figure showed that the prepared GNRs have two bands: a strong 
long-wavelength band at $814 \mathrm{~nm}$ due to the longitudinal oscillation of electrons and a weak short-wavelength band around $508 \mathrm{~nm}$ due to the transverse electronic oscillation, which corresponds to GNRs with an aspect ratio of 3-4. This result is highly congruent with the TEM results. Above all, the GNRs were prepared successfully in this work.

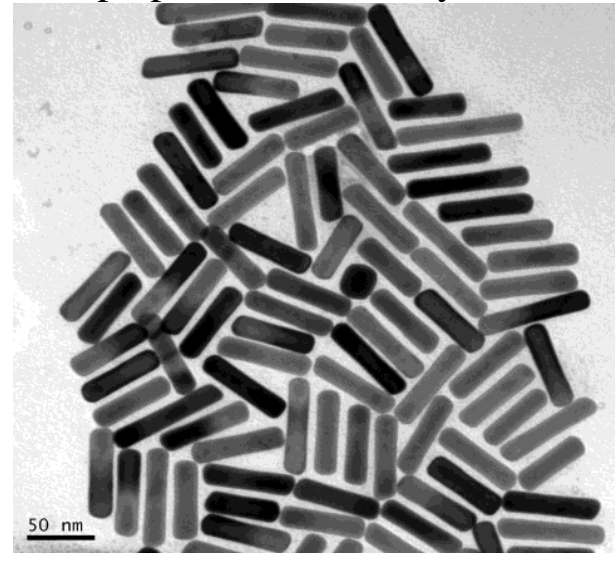

(a)

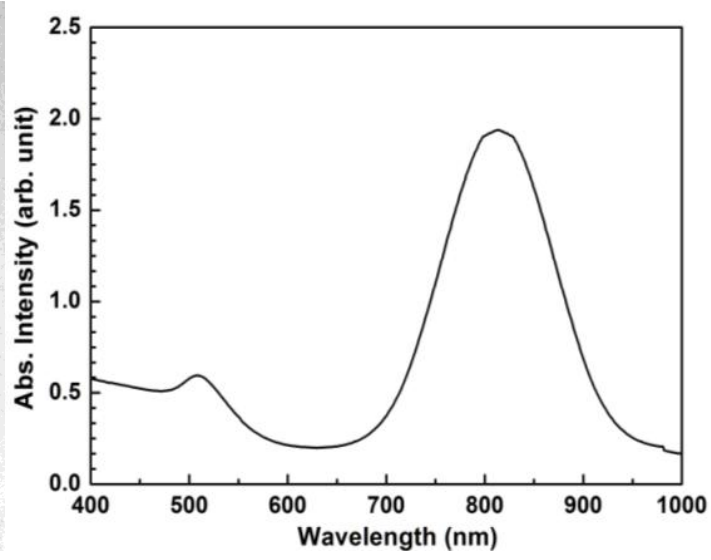

(b)

Fig. 1. (a) TEM image of the prepared GNRs, which are rectangular and round-ended with average transverse diameters of about $20 \mathrm{~nm}$ and longitudinal diameters of $70 \mathrm{~nm}$; and (b) UV-Vis absorption spectra of the prepared GNRs

\section{Characterization of Cur-BSA-NPs, Cur/GNRs-BSA-NPs}

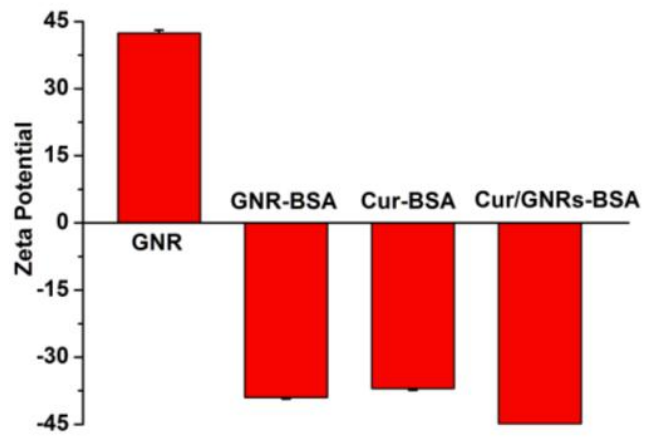

(a)

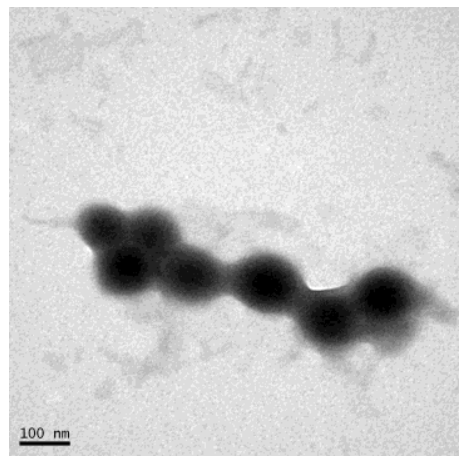

Cur-BSA-NPs

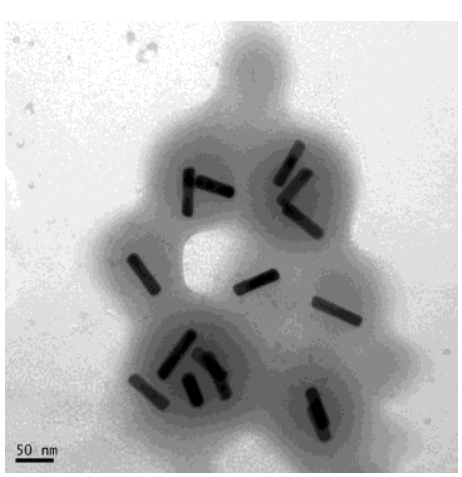

GNR-BSA-NPs

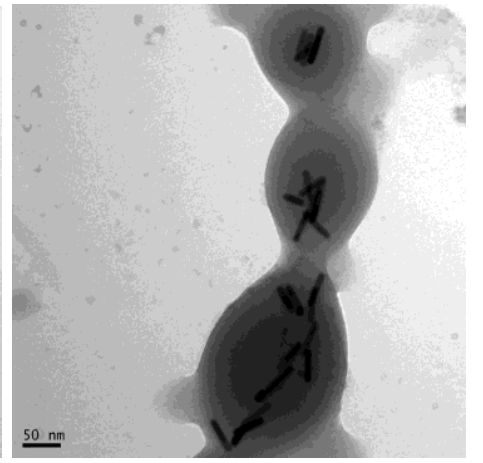

Cur/GNRs-BSA-NPs

(b)

Fig. 2. Fabrication and characterization of CTAB-protected GNR, Cur-BSA, GNR-BSA, and Cur/GNRs-BSA nanoparticles, (a) Surface charge, (b) Representative TEM image

In order to confirm the successful albumin wrapping curcumin and GNRs, the change of surface charge was monitored by zeta-potential measurement. As a blank control, the free GNR displayed positive surface charge of $42.4 \pm 0.7 \mathrm{mV}$ due to the presence of CTAB bilayer. After coating with 
albumin molecules, the surface charge of GNR switched to negative surface charge of $-39 \pm 0.3 \mathrm{mV}$, indicating successful albumin coating on the CTAB outer layer. The surface charge of curcumin wrapped albumin (Cur-BSA) was $-37 \pm 0.4 \mathrm{mV}$ and slightly reduced to $-44.9 \pm 1.1 \mathrm{mV}$ for Cur/GNRs-BSA owning to the GNRs intercalation. As seen in Fig. 3(a), there was no significant change on zeta-potential of the nanoparticles were found after $24 \mathrm{~h}$ storage at room temperature, indicating high stability of the prepared Cur/GNRs-BSA-NPs. The morphology of the nanoparticles was characterized by TEM examination. As shown in Fig. 2(b), the GNR-BSA-NPs dispersed well in aqueous solution as that of parental ones, indicating the change of surface chemistry did not affect hydrodynamic stability of GNRs. The TEM image visually confirmed that GNRs and curcumin were successfully loaded onto the albumin. The average diameters of the Cur-BSA-NPs, GNR-BSA-NPs, and Cur/GNRs-BSA-NPs are $145 \mathrm{~nm}, 140 \mathrm{~nm}$ and $190 \mathrm{~nm}$, respectively, detected by DLS measurement.

\section{Thermodynamic Effect of Cur/GNRs-BSA-NPs}

The Cur/GNRs-BSA-NPs had good hearting effect under NIR light exposure. Fig. 5 shows that the temperature of the prepared Cur/GNRs-BSA nanoparticles could rise to a steady temperature ranging from 38 to $49{ }^{\circ} \mathrm{C}$ when irradiated by NIR laser for $1 \mathrm{~h}$ due to their different concentrations. In the process of heating, the temperature rose rapidly within $30 \mathrm{~min}$, then gently from about $30 \mathrm{~min}$ to 45 min, and stabilized up to a certain level after $50 \mathrm{~min}$. We could also have some conclusion that the temperature rose higher with larger concentration of the nanoparticles. This specific characteristics of hyperthermia was very good for cancer treatment, as it could safely induce tumor cells extinct at a stable temperature $\left(41 \sim 46{ }^{\circ} \mathrm{C}\right)$ while do not harm the normal healthy tissues. We could select suitable temperature for tumor hyperthermia through adjusting the concentration of the prepared Cur/GNRs-BSA-NPs.

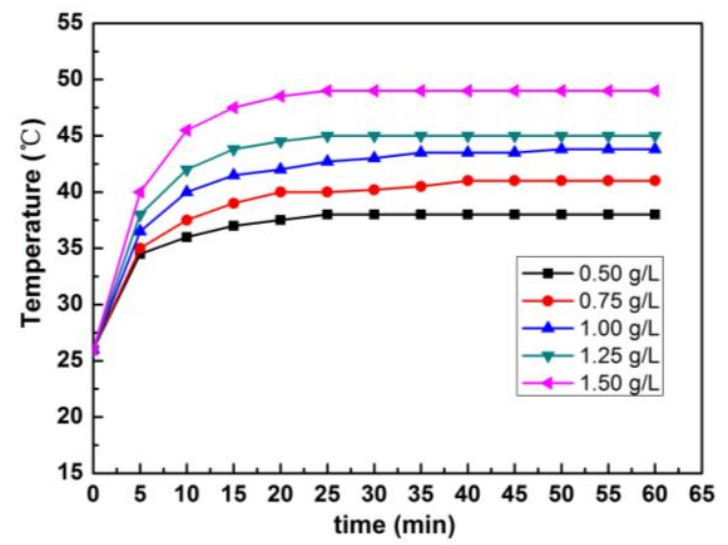

Fig. 3. Temperature elevation of Cur/GNRs-BSA-NPs with different concentrations of $0.50 \mathrm{~g} / \mathrm{L}, 0.75$ $\mathrm{g} / \mathrm{L}, 1.00 \mathrm{~g} / \mathrm{L}, 1.25 \mathrm{~g} / \mathrm{L}$, and $1.50 \mathrm{~g} / \mathrm{L}$ upon NIR irradiation (laser power output of $2.5 \mathrm{~W}$ )

\section{Cytotoxicity of Cur/GNRs-BSA-NPs}

The viability of Cur/GNRs-BSA-NPs treated cells was determined by CCK-8 assay. L929 cells were seeded into 96-well tissue culture plate at a density of $1 \times 10^{4}$ per well in $100 \mathrm{uL}$ of cell culture medium. The cells reached a confluence of $60 \%-80 \%$ after $24 \mathrm{~h}$ incubation. The cells were then incubated with GNR-BSA-NPs, Cur-BSA-NPs or Cur/GNRs-BSA-NPs, as well as a negative group untreated. After another $24 \mathrm{~h}$ incubation, the cytotoxicity of the nanoparticles was evaluated by CCK-8 assay, which was expressed as relative growth rate and calculated as follows: RGR\% $\%$ OD of treated groups / OD of negative untreated group. The results are shown in Table 1. According to RGR, we found that although GNR was very toxic [10], good biocompatibility was found for the GNR-BSA-NPs and Cur/GNRs-BSA-NPs due to albumin modification.

The morphological changes of L929 cells after treatment were also observed by inverted microscopy. They exhibited normal features, such as clear edges, homogenous staining and no cell fragments. The shapes of the treated cells were similar to that of cells in the negative group, which 
was in agreement with the findings from the CCK-8 assay and demonstrated that, coating with albumin molecules, the Cur-BSA-NPs, GNR-BSA-NPs, and Cur/GNRs-BSA-NPs did not show cytotoxicity in vitro.

Tab. 1. The results of cytotoxicity of albumin coating nanoparticles prepared by CCK-8

$$
\operatorname{assay}(\bar{x} \pm \mathrm{S}, \mathrm{n}=6)
$$

\begin{tabular}{cccc}
\hline Groups & Optical Density (OD) & RGR $(\%)$ & Toxicity Grade \\
\hline Negative conrol group & $2.149 \pm 0.221$ & 100 & 0 \\
Cur-BSA-NPs & $2.015 \pm 0.365$ & 93.7 & 1 \\
GNR-BSA-NPs & $1.943 \pm 0.335$ & 90.4 & 1 \\
Cur/GNRs-BSA-NPs & $1.936 \pm 0.212$ & 90.0 & 1 \\
Positive group & $0.421 \pm 0.203$ & 19.5 & 4 \\
\hline
\end{tabular}

\section{Therapeutic Efficiency of Cur/GNRs-BSA-NPs for MCF-7 Cells}

The therapeutic results by the CCK-8 assay are shown in Fig. 4. It could be seen that all the treated experimental groups have proliferation inhibition effects on the MCF-7 cancer cells, except the negative group. The cell growth inhibition efficiency of Cur-BSA-NPs group is about $90.20 \%$ of RGR. This confirms that curcumin could inhibit proliferation of the human cancer cells demonstrated in [11]. While, upon irradiation by NIR light, the GNR was released from GNR-BSA-NPs group, and induced the cancer cells proliferation inhibition with RGR of $79.79 \%$. Due to the thermodynamic advantages of the thermotherapy combined with drug-therapy, the Cur/GNRs-BSA-NPs could significantly inhibit the proliferation of the MCF-7 cells with RGR of 55.66\%. So we could conclude that the prepared Cur/GNRs-BSA-NPs could inhibit proliferation of MCF-7 cells, and may have a preferable therapeutic effect on human breast cancer cells with combined drug and hyperthermia therapy.

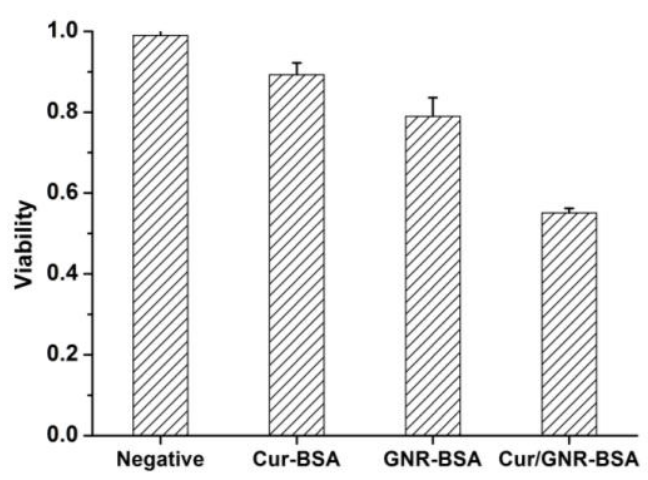

Fig. 4. Proliferation inhibition effect of Cur/GNRs nanoparticles on MCF-7 cells upon NIR laser irradiation by CCK-8 assay

\section{Summary}

Gold nanorod (GNR), an important and classical representative of the metal nanoparticles, has a strong spectra absorption near infrared (NIR) light, causing that subsequent release of energy via heat, which can be used for hyperthermia therapy. This makes it a great potential for medical application. In this work, we successfully prepared the GNRs and Cur/GNRs-BSA-NPs with seedless method and desolvation-crosslinking method, respectively. TEM image showed that the prepared GNRs have uniform sizes and good dispersion. And the GNRs are rectangular and round-ended with average transverse diameters of about $20 \mathrm{~nm}$ and longitudinal diameters of about $70 \mathrm{~nm}$. The prepared Cur/GNRs-BSA-NPs are also approximately spherical and uniform in size with diameter of $150 \mathrm{~nm}$. With the coating of albumin molecules, its cytotoxicity has been greatly reduced tested by CCK- 8 assay in vitro. Thermodynamic test results demonstrated that the temperature of prepared Cur/GNR-BSA-NPs could rise to a steady level of $38 \sim 49{ }^{\circ} \mathrm{C}$ with different concentrations, when 
exposure to the NIR light with wavelength of $808 \mathrm{~nm}$ and output power of $2.5 \mathrm{~W}$. So, the Cur/GNRs-BSA-NPs were suggested to be very useful for tumor hyperthermia. The therapeutic effect on the human breast cancer MCF-7 cells in vitro is tested by CCK-8 assay. The results indicated that the Cur/GNRs-BSA-NPs, when irradiated by NIR laser, could significantly inhibit the proliferation of the cancer cells with the combined drug and hyperthermia therapy. We have not confirmed the mechanism, but this study may provide a new method combining both traditional Chinese and western medical treatment for human breast cancer. However, a lot of work needs to be done if it is to be applied in clinical treatment.

\section{Acknowledgement}

This research was financially supported by the National Natural Science Foundation of China (81171452).

\section{References}

[1] P.K. Jain, I.H. El-Sayed, M.A. El-Sayed, Au nanoparticles target cancer, nano today, 2(1) (2007) 18-29.

[2] E. Molina-Jijón, E. Tapia, C. Zazueta, et al, M. El Hafidi, Z. L. Zatarain-Barrón, R. Hernández-Pando, ... \& J. Pedraza-Chaverri, Curcumin prevents Cr (VI)-induced renal oxidant damage by a mitochondrial pathway, Free Radical Biology and Medicine, 51(8) (2011) 1543-1557.

[3] R. Wilken, M.S. Veena, M.B. Wang, E.S. Srivatsan, Curcumin: A review of anti-cancer properties and therapeutic activity in head and neck squamous cell carcinoma. Mol Cancer, 10(12) (2011) 1-19.

[4] D. Zhao, X. Zhao, Y. Zu, J. Li, Y. Zhang, R. Jiang, Z. Zhang, Preparation, characterization, and in vitro targeted delivery of folate-decorated paclitaxel-loaded bovine serum albumin nanoparticles, International journal of nanomedicine, 5(2010) 669-677.

[5] X.H. Wang, Q.S. Han, J.Y. Li, R. Yang, G.W. Diao and C. Wang, Seedless synthesis of gold nanorods and application in photo-thermal cancer therapy, Acta Phys. -Chim. Sin. 30(7) (2014) 1363-1369.

[6] L. Zhang, S. Hou, S. Mao, D. Wei, X. Song, Y. Liu, Uptake of folate conjugated albumin nanoparticles to the SKOV3 cells, Int J pharm. 287(1-2) (2004) 155-162.

[7] L.K. Zhang, Study on folate receptor-mediated mitoxantrone albumin nanoparticles drug delivery system targeting for ovarian cancer cell, Sichuan University, Chendu, 2004.

[8] L. Li, X.L. Zhao, M.X. Qiao, H.Y. Hu, D.W. Chen, Ch.R. Yang, Preparation optimization of bevacizumab-mediated, doxorubicin-loaded albumin nanoparticles, Journal of Central South Pharmacy, 9 (9) (2011) 641-644.

[9] Y.Y. Yu, S.S. Chang, C.L. Lee, C.C. Wang, Gold nanorods: electrochemical synthesis and optical properties. The Journal of Physical Chemistry B, 101(34) (1997), 6661-6664.

[10] N. Khlebtsov, L. Dykman, Biodistribution and toxicity of engineered gold nanoparticles: a review of in vitro and in vivo studies, Chem Soc Rev, 40 (2011) 1647-1671.

[11] G.H. Di, H.C. Li, Z.Z. Shen, Z.M. Shao, [Analysis of anti-proliferation of curcumin on human breast cancer cells and its mechanism, Zhonghua Yi Xue Za Zhi, 83(20) (2003), 1764-1768. 